\title{
Théories, méthodes et terrains à travers l'étude des trajectoires de vie des ex-enfants soldats en RDC
}

\section{Lucille Grétry}

To cite this article: Lucille Grétry (2015) Théories, méthodes et terrains à travers l'étude des trajectoires de vie des ex-enfants soldats en RDC, Méthod(e)s: African Review of Social Sciences Methodology, 1:1-2, 73-89, DOI: 10.1080/23754745.2015.1017277

To link to this article: https://doi.org/10.1080/23754745.2015.1017277

曲制 Published online: 18 Jun 2015.

Submit your article to this journal ๘

щ Article views: 25

View Crossmark data $\complement$ 


\title{
Théories, méthodes et terrains à travers l'étude des trajectoires de vie des ex-enfants soldats en RDC
}

\author{
Lucille Grétry*
}

\section{Résumé}

Les relations entre théories et terrains ont fait l'objet de nombreux débats en sciences sociales. S'il semble aujourd'hui admis que, pour citer Oliver de Sardan (2008), « c'est dans le rapport au terrain que se joue une part décisive de la connaissance et de l'intelligibilité anthropologique ", quel est donc le rôle de la théorie ? La République Démocratique du Congo est la scène de multiples conflits depuis 1996. L'une des conséquences d'un tel contexte de conflits sur les populations locales est l'enrôlement des enfants dans les groupes armés. À travers l'étude des trajectoires de vie des ex-enfants soldats, comment pouvons-nous comprendre leur passage à l'âge adulte ? Cette question de recherche renvoie à des conceptualisations théoriques aux influences diverses. Deux concepts retiennent ici notre attention : le concept de récit de vie et le concept d'enfance. Dans quelle mesure ces deux concepts ont-ils façonné les différentes étapes de notre travail de terrain ? Mots clés : Enfant soldat ; Militarité ; Récit de vie ; Conflits ; République Démocratique du Congo.

\begin{abstract}
The links between theory and fieldwork have generated countless debates in social sciences. If today we often take for granted that, as Oliver de Sardan (2008) puts it, "it is in fieldwork that we elaborate a determining part of the knowledge and anthropological understanding", what is then the part of theory? Since 1996, the Democratic Republic of Congo has seen multiple conflicts. One of the consequences of a conflict context on local people is the enlistment of children in military groups. Examining the life paths of former child soldiers, how can we fathom their transition into adulthood? This research question brings up several theoretical concepts with various influences. Among these, two concepts hold our attention: life story and childhood. How did these two concepts shape the several stages of our fieldwork?
\end{abstract}

Keywords: child soldier; militarism; life history; conflicts; Democratic Republic of Congo.

\section{Introduction}

Les relations entre théories et terrains ont fait l'objet de nombreux débats en sciences sociales. La théorie, relevant de spéculations abstraites, ne semble pas toujours ancrée au terrain, qui comporte quant à lui une connotation empiriste. S'il semble aujourd'hui souvent admis que, pour citer Oliver de Sardan, « c'est dans le rapport au terrain que se joue une part décisive de la connaissance et de l'intelligibilité anthropologique " (Olivier de Sardan 2008:20), quel est donc le rôle de la théorie dans les recherches contemporaines?

La République Démocratique du Congo (RDC) est, pour reprendre les termes de Mbembe (2000), une postcolonie en crise. Dans ce contexte, la RDC est la scène de multiples conflits depuis 1996. Lune

* FNRS, Pôle Sud, Université de Liège 
des conséquences d'un contexte de conflits sur les populations locales est l'enrôlement d'enfants dans les groupes armés. Le phénomène des enfants soldats, alimentant les débats tant dans le monde de la recherche que dans le secteur de l'humanitaire et du développement, a retenu notre attention et constitué la thématique principale de nos recherches doctorales.

Étudier un phénomène tel que celui des enfants soldats soulève des débats épistémologiques importants. D'abord, quel est le rôle de la théorie pour aborder un sujet de recherche qui se présente à première vue comme particulièrement empirique ? Ensuite, quelles sont les limites des diverses théories et méthodes face au contexte particulier de postcolonie en crise et à un public différent de ceux étudiés par les recherches européennes ? Enfin, pouvons-nous, face à ce type de phénomène, allier théorie, méthode et terrain pour produire de nouvelles connaissances? Dans cet article, nous proposons quelques éléments de réponse à ces questions en nous basant sur nos recherches, menées dans plusieurs provinces de la RDC depuis 2008. À travers l'examen des apports de divers éléments issus de théories ou de méthodes courantes en sciences sociales, telles que l'anthropologie de l'enfance et le récit de vie, nous présenterons d'abord la façon dont notre questionnement de recherche s'est construit. Nous poursuivrons ensuite notre réflexion en discutant du rôle de ces éléments théoriques dans l'élaboration de notre canevas d'enquête. Pour terminer, nous exposerons deux de nos résultats de recherche, choisis précisément pour la manière dont ils interpellent les limites de certains des éléments issus de l'anthropologie de l'enfance et du récit de vie.

\section{La construction du questionnement de recherche...}

La recherche socio-anthropologique est jalonnée de diverses étapes dans lesquelles différentes théories jouent un rôle. Le premier point de cet article est consacré à l'étape de la construction du questionnement de recherche. Nous avons cherché ici à isoler les influences de l'anthropologie de l'enfance d'abord, et du récit de vie ensuite, afin d'exposer quelques-uns des rôles qu'ils jouent dans l'élaboration de notre question de départ.

\section{... à travers l'anthropologie de l'enfance}

Limpact de l'anthropologie de l'enfance sur la construction de notre questionnement de recherche renvoie principalement à trois idées théoriques véhiculées dans le champ, résumées comme ceci : l'enfance est une construction sociale, les enfants sont des acteurs, et les enfants atteignent le statut adulte après des étapes de transition.

Pour commencer, l'enfance est une construction sociale. Les anthropologues de l'enfance, issus de différentes traditions de pensée, et intéressés par divers «types » d'enfance, mettent en évidence le caractère socialement construit de la catégorie d'enfance. À propos de l'enfance et de la jeunesse en général, les anglophones emploient les termes de "social convention " (Hoyles 1979:23). Plus précisément, selon Jenks (1996:61), «l'enfance est une expérience historique et culturelle». Les sociologues et anthropologues francophones rejoignent cette position en parlant de l'" enfant socialement construit " (Sirota 2006) ou en mettant en évidence l'importance d'une définition sociale - et non biologique - de l'enfance (De Boeck et Honwana 2005 ; D'Almeida-Topor et al. 1992). Par ailleurs, l'enfance, loin d'être une catégorie homogène (Lucchini 1996 ; Goodwin-Gill et Cohn 1994), est divisée en divers types et les figures de l'enfance vulnérable (enfant des rues, travailleur, orphelin, sorcier, etc.) ne dérogent pas à la règle du caractère socialement construit (Hart 2006 ; Singer 2005). Par exemple, lorsque Denov (2010) évoque les enfants soldats de Sierra Leone, elle insiste sur la variabilité des définitions de l'enfance et des enfants soldats en fonction de la temporalité et des connaissances contemporaines.

Ensuite, bien que l'anthropologie de l'enfance se soit développée à travers des travaux qui dans un premier temps, ont abordé la construction sociale et symbolique de l'enfance à partir des discours tenus par les adultes sur les enfants, les travaux contemporains, sous l'impulsion des concepts d'« enfant- 
acteur » et d'agency, s'éloignent de la conception de l'enfant comme être passif, vulnérable, innocent ; ils s'inscrivent dans la reconnaissance de celui-ci en tant qu'acteur social (Boyden 1997 ; Muxel et Cacouault 2001) doté de marges de manœuvre (Danic et al. 2006), de capacité d'action et d'autonomie dans ses décisions (Machel 1996). De Trefon (2004) qui met en évidence le poids démographique de la jeunesse, à De Boeck (2005) qui considère les enfants comme de véritables forces sociales émergentes par la place centrale qu' ils occupent dans les espaces publics, les anthropologues s'accordent sur le statut d'acteur social de l'enfant africain. Les figures de l'enfance vulnérable n'échappent pas à la grille de lecture de l'agency. Ainsi, tour à tour les systèmes normatifs produits par les enfants des rues (Tessier 1995, Darbon et du Bois de Gauduson 1997), leur volonté de maitriser leur réinsertion (de Suremain 2006, Fall et Massart 2007), leur influence dans la construction politique et socio-économique (De Boeck 2005), et enfin leur pouvoir de décision quant aux choix stratégiques de mode de survie (Tatek 2008 ; Gretry 2011), seront mis à jour. Cependant, la figure de l'enfant soldat, plus ambiguë, témoigne de capacités d'action spécifique (Honwana 2000) et les théories de l'agency sont loin d'avoir le monopole de l'explication de ce phénomène. Deux grilles de lecture se superposent alors : d'un côté, une perspective structuraliste explique la participation des enfants dans les conflits armés par des structures et des forces qui leur sont extérieures ; de l'autre, des analystes intègrent le concept d'agency à leurs travaux et suggèrent que les enfants soldats agissent sur la base de choix rationnels (Denov 2010).

Pour terminer, nous pensons pouvoir imputer un troisième élément théorique à l'anthropologie de l'enfance : une transition sépare « deux âges » (Garnier 1995). En effet, les différents auteurs cités jusqu’à présent parlent de l'âge de l'enfance et de l'âge adulte, le premier précédant le second. Les recherches en socio-anthropologie de l'enfance se sont ainsi intéressées à l'intégration progressive des enfants dans le monde des adultes par des rituels permettant leur passage d'une classe d'âge à une autre. Aujourd'hui, l'idée de transition (Goodwin-Gill et Cohn 1994:6-7 ; Singer 2005:7 ; Biaya 2001:29 ; Neyzi 2001:102) ou, pour le dire autrement, d'une entrée dans la vie adulte (Antoine et al. 2001:17) n'est pas questionnée. Cependant, les critères de démarcation entre les deux catégories, eux, suscitent la réflexion. Nous partons, en Afrique subsaharienne, de sociétés où l'âge social, sous l'angle de la génération et de la séniorité sociale, politique et sexuée, était structurant d'une manière forte et très particulière, pour aboutir à une situation contemporaine d'anomie entre d'anciens modèles en décomposition et un projet de modernité qui a échoué. En effet, « la complexification des conditions d'insertion sociale définit de nouveaux modes de socialisation et participe au déclin des formes traditionnelles du passage à la vie adulte " (Muxel et Cacouault 2001:68). Face aux réalités nouvelles, d'une part, les jeunes prendraient et assumeraient précocement des rôles et des fonctions (Fall et Massart 2007) et, d'autre part, le statut d'adulte deviendrait financièrement inaccessible (De Boeck 2005:10). Dès lors, dans ces contextes, l'idée de transition entre deux âges mérite une attention accrue. Les anthropologues situent de nouveau ici les enfants soldats dans une position particulière par rapport aux autres figures de l'enfance vulnérable. La possession d'armes et le droit de tuer les placeraient en dehors de la catégorie des enfants, mais ne suffiraient pas à les intégrer pleinement dans la catégorie des adultes (Kuyu 2008). Ils occuperaient ainsi « des espaces sociaux interstitiels, entre les mondes adulte et juvénile (Honwana 2000:58). Les travaux s'intéressant avant tout à la manière dont on fait de l'enfant un adulte, ont été critiqués pour avoir abordé l'enfant dans son devenir, en tant qu'être incomplet, immature, à construire ou à éduquer. Cependant, cette démarche et celle de l'étude de l'enfant dans son temps présent ne nous semblent pas être des perspectives contradictoires mais plutôt complémentaires.

Les trois éléments théoriques exposés ci-dessus ont chacun joué un rôle dans l'élaboration de notre questionnement de recherche. D'abord, la notion de construction sociale de l'enfance nous a poussée à interroger les définitions et représentations sociales de l'enfance et des enfants soldats. Ensuite, l'appréhension de l'enfant comme acteur social a suscité des investigations au sujet de ses stratégies, pratiques et prises de décisions. Enfin, l'idée de transition à un statut d'adulte a engendré une réflexion sur les modalités de passage à l'âge adulte. 


\section{...à travers le récit de vie}

La théorie ou méthode du récit de vie, au confluent de diverses disciplines des sciences humaines, a également joué un rôle dans l'appréhension de notre thématique. Du récit de vie (Bertaux 1997; Burrick 2011 ; Demazière 2003 ; Poirier et al. 1989) à l'histoire de vie (Bourdieu 1986 ; Delory-Momberger 2005 ; Lainé 2008 ; Le Grand 2000 ; Pineau et Le Grand 1993) en passant par le parcours de vie (Guillaume et al. 2005), la terminologie des enquêtes biographiques est variée et les définitions des concepts sont subtiles. Pour la clarté du texte, nous avons retenu le concept de récit de vie. Malgré la diversité des termes et des différentes dimensions, la théorie ou méthode du récit de vie a influencé notre questionnement de recherche et ce, à partir de trois notions : la subjectivité du récit, l’identité dans le récit, et la temporalité à travers le récit.

Tout d'abord, lorsque les auteurs définissent les concepts de l'enquête biographique, ils se positionnent par rapport à la construction de l'histoire faite par celui qui se raconte. Pineau et Le Grand (1993:5) insistent sur l'histoire de vie, vaste champ dans lequel le récit de vie s'inscrit, en tant que « construction de sens ». Legrand (1993), lui, oppose l'histoire de vie, objective, au récit de vie, technique de recueil de données, narration ou récit. Bertaux (1997), plus souple, définit le récit de vie comme la forme narrative d'une production discursive, et l'histoire de vie comme la reconstruction a posteriori d'une cohérence, dont le chercheur est conscient. De son côté, Bourdieu (1986:70), à ce propos, met en évidence l'illusion biographique produite par l'activité narrative, au sens où traiter de la vie "comme le récit cohérent d'une séquence signifiante et orientée d'évènements ", est une illusion. Lainé (2008), pour terminer par lui, considère le récit de vie comme l'expression d'une médiation entre la vie et l'histoire de vie. Ainsi, le concept ne fait pas l'unanimité auprès des auteurs qui s'interrogent quant à la subjectivité du récit de vie.

Ensuite, les auteurs mettent en évidence l'importance de la question de l'identité, au coeur du récit de vie. Quand ils transmettent leurs récits de vie, les individus explorent ce qu'ils sont devenus (Lévesque 2003), deviennent auteurs de leurs histoires (De Gaujelac 1987), et construisent leurs identités narratives (Ricoeur 1985) ou leurs savoirs biographiques (Delory-Momberger 2005).

Enfin, dans la définition du récit de vie, la notion de temporalité est récurrente chez la plupart des auteurs. De Demazière et Dubar (2009), pour qui « faire son récit de vie consiste à articuler la temporalité avec la mise en mots ", à Delory-Momberger (2006:14), qui écrit, plus largement à propos du discours biographique, qu'il revient à " configurer narrativement la succession temporelle de son expérience ", les auteurs, quelle que soit la terminologie qu'ils emploient et la place qu'ils accordent à la question de la subjectivité ou de l'identité, s'entendent sur l'existence d'une dimension temporelle, apparemment intrinsèque aux récits de vie. Burrick (2010), dans son épistémologie du récit de vie, érige d'ailleurs l'étude de la temporalité au rang de spécificité du récit de vie. Plus loin encore, Bertaux (1997) va jusqu'à identifier deux axes temporels : la diachronie, ou la succession temporelle des évènements, et la chronologie, c'est-à-dire la datation en termes d'années ou d'âges. Enfin, Guillaume, Lalive d'Epinay et Thomsin (2005), dans le paradigme des parcours de vie, donnent à la question du temps, dont la durée caractérise les étapes de vie, une position centrale qu'ils déclinent en deux composantes : la temporalité de l'individu et le temps historique.

Ainsi, notre questionnement de recherche a été façonné par les concepts issus du récit de vie présentés ci-dessus. La question de la subjectivité du récit nous a engagée à interroger les liens entre les évènements objectifs de la vie des enfants soldats, et le récit qu’ils en font. La place accordée à l'identité dans le récit nous a suggéré d'approfondir la question de l'identité des enfants soldats. Enfin, l'importance de la temporalité dans le récit nous a invitée à interroger la dimension temporelle, en tant que configuration première du récit des expériences de vie, et ainsi à investiguer les stratégies, pratiques et étapes de vie des enfants soldats dans leur temporalité, tant diachronique que chronologique. 


\section{Pour aboutir à une question de départ}

Les apports conjugués des éléments théoriques issus de l'anthropologie de l'enfance et du récit de vie ont abouti à la question de départ suivante : comment les enfants soldats deviennent-ils adultes en RDC ? Pour y répondre, nous nous proposions premièrement de circonscrire les termes d'enfant soldat et d'adulte à travers l'étude combinée des représentations sociales locales de l'enfance et des enfants soldats, des dimensions de l'identité des deux statuts et des modalités de passage d'un état à un autre. Le décor planté, nous nous devions alors d'entrer dans le vif du sujet en nous intéressant aux pratiques et stratégies mises en place par les enfants soldats pour conquérir le statut d'adulte, tout en examinant la distance que prennent leurs récits par rapport aux faits objectifs et à la temporalité de leurs étapes de vie.

\section{L'élaboration du canevas d'enquête...}

Dans cette deuxième section, nous nous attardons dans un premier temps sur les apports del'anthropologie de l'enfance pour ensuite orienter notre réflexion vers ceux du récit de vie dans la préparation du canevas d'enquête.

\section{... à travers l'anthropologie de l'enfance}

Les éléments théoriques issus de l'anthropologie de l'enfance et relatifs à l'élaboration de notre canevas d'enquête ont joué un rôle à deux niveaux : la définition de l'échantillon de recherche et l'élaboration des outils de récolte de données.

Commençons par la définition de l'échantillon de recherche. Comme nous l'avons évoqué dans le premier point, l'enfance est une construction sociale. Certains chercheurs poussent cette idée jusquà utiliser une définition sociale de l'enfance pour construire leur échantillon de travail. C 'est le cas de Danic, Delalande et Rayou (2006:13), selon lesquels le critère permettant d'identifier des individus comme des enfants est de "savoir s'ils sont considérés comme tels par le groupe social choisi pour objet d'étude ». Nous avons suivi leur exemple et nous sommes référée à la définition sociale des enfants soldats pour construire notre échantillon. Afin de connaître cette définition, nous avons mis au point un jeu de classement, comme support préalable à la discussion. L'exercice se fonde sur l'idée que la perception de l'autre s'organise à travers des catégories, duelles ou multiples. L'enquêteur propose une série de portraits aux enquêtés et leur demande de classer ces portraits en groupes. Concrètement, nous avons utilisé une série de 16 images $^{1}$, testées préalablement afin de déceler les éventuelles ambiguïtés contenues dans certains portraits. Prenons un exemple concret. L'un des portraits que nous utilisions dans cette phase exploratoire était issu d'un film. Les " enquêtés-test " ne l'ont pas reconnu, nous avons donc conservé cette image dans la phase ultérieure de récolte de données. Au final, la série de 16 portraits retenue comprenait à la fois des hommes et des femmes; des blancs et des noirs ; des personnes armées, en uniforme ou pas, et des personnes à première vue civiles; et enfin des adultes, des enfants et des personnes âgées. Les personnes interrogées, principalement des intervenants locaux dans le secteur de la protection de l'enfance ou des parents d'ex-enfants soldats, devaient classer chacun de ces portraits sans exception, dans le nombre de groupes qu'ils souhaitaient. Dès lors, employer le terme " ex-enfants soldats ", qui correspond aux catégories langagières spontanées de la population congolaise pour parler de ceux que les organisations internationales nomment les " enfants sortis des forces et groupes armées (ESFGA) ", n'émane pas du hasard. Selon la définition qu'ils donnent d'eux-mêmes, très proche des critères que les professionnels de la protection de l'enfance leur assignent - ce que nous avons découvert dans le jeu de classement et de nombreuses discussions informelles - les personnes éligibles à notre échantillon de recherche devaient répondre à deux caractéristiques : avoir intégré un groupe armé, et l'avoir fait avant l'âge de 18 ans. Nous décelons ici l'influence de la Convention Internationale des Droits de l'Enfant (CIDE) qui définit l'enfant comme toute personne de moins de 18 ans. Cette limite chronologique montre que la représentation de l'enfance et des enfants soldats est liée très directement aux textes légaux 
qui entourent les mécanismes de protection. En effet, ce critère de l'âge est cité par toutes les personnes que nous avons rencontrées lors de nos recherches de terrain, que ce soit de manière informelle, dans des entretiens plus cadrés ou dans le jeu de classement, et souvent en référence à la CIDE. Ainsi, l'élément principal de définition de l'enfant soldat provient du registre du droit international. Lidéologie est communiquée d'abord aux intervenants congolais, convaincus en principe, par l'intermédiaire de multiples formations; et ensuite aux populations locales, par diverses séances de sensibilisation. Notons toutefois que si la limite chronologique est bien effective pour définir l'enfance à partir de critères locaux dans le cas de la RDC, ce type de définition est remis en question par les chercheurs partisans d'une approche constructiviste de l'enfance, qui mettent en exergue des délimitations sociales, et qui qualifient d'arbitraire la délimitation de l'enfance à un âge biologique précis (Boothby et al. 2006:13; Rosen 2005; Twum-Danso 2005:11). En suivant le critère d'intégration dans un groupe armé en deçà de 18 ans quel que soit l'âge présent, nous pouvions inclure dans notre échantillon des personnes ayant incorporé des groupes armés gouvernementaux autant que rebelles, et y ayant exercé de multiples fonctions. Au niveau de la répartition, 71 personnes dont 55 hommes et 16 femmes composent notre échantillon de recherche. La faible proportion de femmes s'explique d'une part par la masculinité encore très présente du métier de soldat, d'autre part par le refus des chefs de groupes armés de démobiliser les jeunes filles (considérées comme des fiancées, des cuisinières, des lessiveuses, etc. et non pas comme des soldats), et enfin par la stigmatisation des femmes sorties des groupes armés, plus puissante que celle dont sont victimes les hommes, les rendant ainsi plus réticentes à affirmer leur statut d'anciens soldats et à répondre à une enquête basée sur ce passé. Par ailleurs, 43 personnes ne sont plus soldats au moment où nous les avons interrogées, ce qui ne signifie pas pour autant qu'elles aient été préalablement démobilisées de manière officielle. Pour les autres, 13 membres de notre échantillon sont militaires, 9 sont en centre de transit et d'orientation ou en centre d'accueil et donc très récemment sorties d'un groupe armé, et enfin 6 possèdent un statut ambigu (ils sont policiers, blessés, ou vivent au camp sans se considérer comme des militaires). Ainsi, la distance temporelle et spatiale d'avec le monde militaire est extrêmement variable d'un individu à un autre de notre échantillon. Du point de vue géographique, nous avons interrogé 36 personnes à Kinshasa, 3 à Matadi, 7 à Mwanda, 12 à Lubumbashi et 13 à Goma. En ce qui concerne l'âge des individus interrogés, toute personne ayant été enrôlée à moins de 18 ans, quel que soit son âge actuel, était éligible à notre échantillon. Dès lors, les personnes composant notre échantillon se sont enrôlées entre 8 et 17 ans à des périodes très diverses. En conséquence, elles avaient entre 13 et 33 ans lorsque nous les avons rencontrées. Soumettre à la passation d'un entretien de type classique une personne de 12 ou de 33 ans ne constitue pas le même exercice. Pour les plus jeunes, la tâche est rude. Que ce soit à cause d'un temps de concentration plus court, de la difficulté de comprendre l'acceptabilité de toutes les réponses, ou encore de la présence des parents, procéder à l'entretien semi-directif des plus jeunes personnes de notre échantillon de recherche n'a pas produit un grand nombre de données. Face à la pauvreté de nos données les concernant, nous avons alors utilisé un support projectif pour trois des plus jeunes enquêtés : des planches de bandes dessinées. Après les avoir regardées, les jeunes enquêtés recevaient simplement la consigne de parler de ces images. Ces supports ont véritablement déclenché leur récit car, plus que de parler des planches telles quelles, ils y projetaient leur propre histoire. L'extrait d'entretien suivant, réalisé à Lubumbashi auprès d'une jeune sortie d'un groupe rebelle moins de 6 mois auparavant, témoigne du transfert que font les enquêtés de leur propre histoire sur les planches de bandes dessinées :

"Là, c'est de la même façon que quand on nous avait pris. Nous étions devant, il y avait un autre militaire derrière, vous avancez, si vous essayez de bouger, vous risquez d'attraper la balle, il peut tirer sur vous. C'est comme ça, quand on nous arrêtait. Si tu oses fuir, il tire sur toi et tu meurs. Puis les autres passent et continuent la marche. Si lui se tient devant, tu risques de fuir. C'est pourquoi il préfere être derrière pour surveiller tout le monde. Ce ne 
sont pas des soldats, ça ressemble au moment où on nous prenait nous, et on nous amenait quelque part. » [Rachel]

Ainsi, accepter comme définition de la population cible des critères locaux est un choix méthodologique. Cette option a l'avantage d'ancrer profondément le chercheur sur son terrain. Cependant, elle engendre le risque de devoir composer l'échantillon de recherche de profils multiples. Dans ce cas, le chercheur se verra contraint d'utiliser des outils de récolte de données variés correspondant aux diverses caractéristiques de son échantillon, tout en s'assurant que la rigueur de son travail ne puisse être remise en cause.

Poursuivons la création proprement dite des outils de récolte de données. Dans la perspective de l'enfant acteur, de nouvelles recherches « se fondent sur une méthodologie mettant au centre l'enfant comme interlocuteur de l'enquête" (Danic et al. 2006:52). Dans ce sens, les recherches de Denov à propos de la vie des enfants soldats de Sierra Leone aboutissent à des résultats étonnants, produits notamment grâce à une méthodologie basée sur une approche participative où des ex-enfants soldats ont été engagés comme chercheurs et collaborateurs. En nous inspirant de cette méthode et en gardant en tête l'intérêt de placer l'enfant au centre de la recherche, nous avons mis au point un nouvel outil de récolte de données : l'exercice " enquêteur " - « enquêté ». L'exercice s'opère par la réalisation d'un entretien d'une personne de la population cible par une autre personne elle-même issue de la population cible. Devenir enquêteur et procéder à une démarche en sciences sociales ne s'improvise pas, mais s'apprend et se travaille. Il ne s'agit donc pas ici de placer nos enquêtés dans le rôle d'un chercheur à part entière. Plus modeste, l'objectif de l'exercice est de les investir d'une fonction de " poseur de questions ", ce qui implique qu'ils les choisissent, les posent effectivement, et les approfondissent s'ils le souhaitent. Concrètement, dans notre recherche, 16 ex-enfants soldats se sont prêtés au jeu et sont devenus l'espace d'une petite heure des " enquêteurs " ou des " enquêtrices " interrogeant des ex-enfants soldats et/ou des enquêtés questionnés par des ex-enfants soldats. Chaque enquêté(e) avait déjà vécu une situation d'entretien de type classique auparavant. À leur arrivée pour un second rendez-vous, ils savaient qu'ils rencontreraient d'autres personnes participant à la même recherche. Après leur avoir donné les consignes de l'exercice, nous laissions l'enregistreur sur la table, et nous installions de l'autre côté de la pièce. Si le démarrage a parfois été difficile, les " enquêteurs » et enquêtés ont vite pris leur rôle au sérieux. Bien qu'il soit conseillé de ne jamais limiter la durée d'un entretien, nous avons dû, face au risque de n'obtenir que des enregistrements superficiels de quelques minutes, nous résoudre à imposer une durée de 30 minutes aux enquêtés pour les encourager à approfondir l'entretien. Les questions méthodologiques relatives à la mise en place de cet outil sont nombreuses. D'abord, la présence du chercheur mérite d'être questionnée. Si elle engendre un biais non négligeable, des raisons matérielles, d'organisation et de confiance, ne laissent pas toujours le choix au chercheur qui se doit alors de s'effacer au maximum afin de minimiser les biais que sa présence induit. Ensuite, la passation d'un entretien de type classique avant l'exercice " enquêteur » - " enquêté » suscite également le débat, car elle peut avoir pour conséquence d'influencer les questions que les enquêtés se poseront par la suite. Nous avons choisi cette option car une première expérience d'entretien a semblé nécessaire aux enquêtés pour répondre à la consigne de l'exercice " enquêteur » - « enquêté ». Malgré les questions méthodologiques relatives à la mise en place de l'outil, que le chercheur se devra de garder en tête lors de l'interprétation de ses résultats de recherches, les apports de l'exercice " enquêteur » - " enquêté " comparativement à l'entretien classique sont multiples et se situent tant dans la forme de l'entretien que dans son contenu. En ce qui concerne la forme, nous dénombrons trois types de contributions de l'exercice " enquêteur " - « enquêté ». D'abord, l'exercice localise les effets des biais de recherche tout en diminuant les effets secondaires, et la circonscription de ces biais nous permet de les corriger, ou, si ce n'est pas possible, d'interpréter nos résultats en parfaite connaissance de cause. Ensuite, si la création d'un climat de confiance et l'appropriation d'un savoir commun par une population cible sont des processus lents et non exhaustifs, l'exercice " enquêteur »" enquêté » résout en partie ce problème. En effet, l’apprenti « enquêteur ", un pair, connait les limites 
dans lesquelles il peut se permettre de questionner son enquêté, et ce dernier est parfois plus enclin, sur certaines dimensions, à se dévoiler face à un inconnu semblable plutôt que face à un inconnu différent. Ainsi, l'exercice « enquêteur » - « enquêté » solutionne partiellement une difficulté inhérente à une enquête socio-anthropologique en milieu étranger en éclairant de données certaines des "zones " qui seraient laissées "blanches" (Bertaux 1997) par des entretiens de type classique. Enfin, l'exercice " enquêteur " - " enquêté » inverse le rapport de domination habituel. En effet, dans les entretiens de type classique, la population cible figure dans la position de dominé (Danic et al. 2006). Dès lors, « le chercheur et l'objet de la recherche sont souvent répartis dans des catégories puissant/impuissant où le chercheur exerce un pouvoir sur l'objet de la recherche " (Thapar-Bjorket et Henry 2004 ; cité par Denov 2010:80). En donnant la place d'enquêteur aux ex-enfants soldats issus de l'échantillon, l'exercice " enquêteur » - « enquêté » présente l'avantage de pouvoir inverser ce rapport de domination. L'apprenti " enquêteur ", alors dans une position qui se rapproche de celle de dominant, s'autorise à aborder les thèmes qu'il souhaite.

En ce qui concerne le contenu des données, les apports de l'exercice « enquêteur » - « enquêté » sont également divers. D'abord, deux éléments - être un outil différent de l'entretien classique et être utilisé après ce même entretien classique - s'agencent pour produire in fine des données supplémentaires. Ensuite, l'exercice " enquêteur »- « enquêté » permet à la population cible de s'exprimer en ses termes, et ainsi de dévoiler les catégories langagières locales.

Enfin, l'idée d'une transition entre l'enfance et l'âge adulte nous invite à considérer les enfants comme un groupe social à part entière, distinct de celui des adultes. En conséquence, il nous a semblé indispensable d'adapter les outils de récolte de données conçus pour des adultes au caractère particulier de l'enfant, pour les plus jeunes de nos enquêtés. Nous avons dès lors mis au point une variante du focus groupe : le focus sur base de dessins. Le focus groupe, procédure de collecte de données, fournit des informations sur ce que les gens pensent ou ressentent (Krueger 1994). Le focus est enclenché par une consigne de départ de la part de l'enquêteur. S’en suivra alors une directivité ou semi-directivité de ce dernier. Dans le cas du focus sur base de dessins, l'exercice démarre par un dessin réalisé par les enquêtés et l'explication qu'ils en donnent. L'objectif de ce type particulier de discussion collective est double : baser la discussion sur une production des enquêtés et proposer un support de données considéré comme adapté aux enfants. Ainsi le focus sur base de dessins est moins directif que le focus groupe classique ; il autorise notamment les enquêtés à s'interroger mutuellement sur les dessins et l'explication donnée. Concrètement, nous avons organisé, au cours de nos enquêtes de terrain, 21 focus sur base de dessins nonmixtes, regroupant chacun entre 2 et 4 enquêtés. 26 personnes ont participé à deux focus sur base de dessins, et, pour des raisons matérielles, 8 personnes n'ont participé qu'à une seule séance. Les participant(e) s, pour la plupart, avaient déjà été rencontrés lors de passations d'entretiens individuels, ou d'observations dans des centres de transit pour enfants soldats. Parmi les 34 personnes ayant pris part aux focus sur base de dessins, nous comptons 7 filles et 27 garçons. Les focus sur base de dessins commençaient par l'énoncé d'une consigne très large. Puis, les enquêtés disposaient du temps nécessaire à la confection de leur dessin et s'interrogeaient ensuite les uns et les autres à propos de chaque dessin. Seulement après cette première partie de discussion, nous intervenions pour approfondir certains points cités préalablement. Tout comme la mise en place de l'exercice " enquêteur " - " enquêté ", celle du focus sur base de dessins pose question. D'abord, bien que l'outil se veuille moins directif qu'un focus groupe classique, l'orientation du dessin sera fonction de l'énoncé donné par l'enquêteur qui se doit alors de travailler au maximum la diminution du biais introduit par ce même énoncé. Par ailleurs, l'enquêteur doit être conscient que le copiage des enquêtés les uns sur les autres a des conséquences. Enfin, l'outil présuppose que le dessin est adapté à la recherche avec des enfants. Or, le caractère approprié du dessin, que ce soit pour les enfants ou pour les adultes, peut être fonction de la culture de la population cible. 


\section{... à travers le récit de vie}

Dans l'élaboration du canevas d'enquête, le récit de vie a, lui aussi, influencé notre méthodologie. Comme nous l'avons évoqué précédemment, la théorie ou méthode du récit de vie met en évidence la structuration de ce récit de vie autour "d'une succession temporelle d'évènements et de situations " (Bertaux 1997:33). Au niveau de notre canevas d'enquête, cet élément théorique a imprégné de manière diffuse l'ensemble de notre processus de recherche en nous poussant à rechercher dans nos observations, mais encore plus dans nos passations d'entretiens semi-directifs, des indicateurs temporels. Ainsi, notre première grille d'entretien était basée sur une structuration temporelle des évènements au moyen de l'utilisation d'indicateurs temporels pour guider l'échange.

Ensuite, selon la plupart des auteurs, le récit de vie est une narration. De Legrand (1993:180) qui le définit comme « la narration ou le récit, écrit ou oral, par la personne elle-même de sa propre vie ", à Kaufmann (2004:157), qui nous parle de "fil narratif » mis en place par le sujet en passant par Orofiamma (2002:3), selon qui « toutes les pratiques qui ont recours aux récits biographiques ont en commun de s'appuyer sur [...] la narration ", la configuration du récit de vie semble être uniformément conçue comme narrative. S'inscrivant dans l'approche narrative du récit de vie, l'enquêté devient le "narrateur " (Poirier et al. 1989:75) qui, en se racontant, se met en scène dans des scénarios (Orofiamma 2008). À propos du discours narratif, notons que d'une part, il n'exclut pas l'insertion d'autres formes de discours en son sein (Bertaux 1997) ; que d'autre part, il recourt à deux registres de données : des événements (ou réalité objective) et des significations (ou réalité subjective) (Orofiamma 2008). Revendiquer la dimension narrative du récit de vie permet de déceler un ensemble de règles auxquelles les récits obéissent. Tout d'abord, le discours narratif engage à la cohésion de la vie et fait du récit une totalité hautement organisée. Ensuite, le travail de la narration invite à élaborer une histoire parmi d'autres histoires possibles. Enfin, le discours narratif est une expérience qui permet de se positionner par rapport aux autres (Orofiamma 2002). Déployant l'approche narrative du récit de vie, Bertaux (2997:58) propose une forme particulière d'entretien pour le recueillir : "l'entretien narratif ». Cet entretien doit être lancé par une phrase contenant le verbe " raconter ». Sa conduite est cruciale, car c'est dans cette situation de communication et d'interaction avec le chercheur, traversée par des attentes, des enjeux de pouvoir et de reconnaissance, que seront produits la forme du récit (Orofiamma 2002) et le code narratif (Demazière et Dubar 2009). Sous l'influence de l'importance du discours narratif dans le récit de vie, nos entretiens semidirectifs se sont voulus des entretiens narratifs.

\section{Pour aboutir à une méthodologie particulière}

Nous avons défini notre échantillon de recherche selon les définitions et les représentations sociales locales de l'enfance et des enfants soldats, elles-mêmes saisies au moyen d'un outil méthodologique original : le jeu de classement. Ensuite, une fois accepté(e) s dans l'échantillon de recherche, les enquêté(e) s répondaient à un ou deux premiers entretiens narratifs semi-directifs, imbibés d'indicateurs temporels. Les premières rencontres avaient lieu dans des espaces publics, calmes et isolés, choisis par nos soins avec l'aide de nos interprètes et selon la mobilité de l'enquêté(e). Après quoi, si l'enquêté(e) le souhaitait et que son domicile s'y prêtait, nous nous y rendions pour le second rendez-vous. Après ce ou ces premiers entretiens, les enquêté(e) s se rencontraient alors pour participer à un exercice " enquêteur " - "enquêté " et/ou à un ou deux focus sur base de dessins, dans des salles de réunion mises à notre disposition au sein de quartiers faciles d'accès dans les différentes villes dans lesquelles nous avons investigué. Ainsi, nos analyses se basent sur un cumul de témoignages récoltés à des moments et en des lieux distincts, à partir d'outils méthodologiques variés. Les circonstances de production de récits ont donc été multiples. 


\section{La présentation des résultats...}

Les résultats auxquels nos recherches ont abouti sont multiples. Deux d'entre eux ont la particularité d'effleurer les limites de l'anthropologie de l'enfance et du récit de vie. Dans la troisième et dernière partie de ce texte, nous allons nous atteler à exposer ces résultats, ainsi que la manière dont ils ébranlent les deux courants.

\section{La dualité civil/militaire vs enfancelâge adulte}

Comme nousl'avons expliqué, notre questionnement de recherche se donnait pour objectifd'appréhender la transition du statut d'enfant soldat à celui d'adulte en RDC. La question de la transition d'un statut à un autre nous semble supposer l'existence de ce que nous nommons ici la dualité enfance/âge adulte. Or, la présence de cette dualité enfance/âge adulte, ou plus précisément sa prépondérance dans le parcours de vie d'un individu, ne va pas forcément de soi. Nos premiers résultats ont en effet mis en évidence le manque d'effectivité de la dualité enfance/âge adulte, et, par opposition, l'importance de la dualité civil/ militaire. Nous allons analyser le « désenracinement » de la dualité enfance/âge adulte comme point de repère du parcours biographique d'un individu à travers l'expérience de vie des ex-enfants soldats et les représentations sociales des professionnels de la protection de l'enfance.

Commençons par l'expérience de vie des ex-enfants soldats. D'abord les relations qu'ils entretiennent avec les adultes soldats au sein des groupes armés témoignent du manque de séparation nette entre les deux catégories. Si une minorité d'ex-enfants soldats rapporte une relation de type paternaliste, et parfois en termes négatifs, l'analyse des entretiens met en évidence une relation majoritairement égalitaire, exprimée de manière positive, entre enfants et adultes soldats. Laissons Lwanda, un ex-enfant soldat interrogé en octobre 2010, vivant au Katanga, prendre la parole :

"Avec les militaires plus âgés, tout se faisait de la même façon. Si vous allez à la guerre, vous allez combattre tous de la même façon. Dans le combat, on ne peut pas dire que celui-ci est grand, celui-ci est petit. S'il y a des tâches à vous confier, on vous les confie sans distinction ». Ensuite, l'étude des récits de vie des ex-enfants soldats nous apprend que les notions d'âge, d'aînesse, ou de séniorité, comme principes communs du politique (Abélès et Collard 1985) et de l'inégalité sociale (Bayart 1990), ne sont pas des réalités intrinsèquement liées à la date de naissance d'une personne. Être grand en RDC peut revêtir plusieurs significations. Et dans les groupes armés, le grade confere sa grandeur au soldat qui le possède. John, un militaire de Kinshasa enrôlé dans l'Est du pays à l'âge de 14 ans, nous dit à ce propos que : "Nous sommes mélangés. Dans l'armée, il n’y a pas les plus âgés. Si vous êtes plus gradés, c'est plus âgé dans l'armée. Même si tu es petit, tu peux diriger un papa qui a l’âge peut-être de 45 ans si tu es plus gradé que lui. Dans l'armée il n'y a pas de plus âgé». Plus généralement, nos analyses mettent en exergue les bouleversements de l'organisation hiérarchique d'un groupe militaire, par rapport à la société civile. Les jeunes enfants occupent, en RDC, une position de soumission face à leurs parents ou à leur tuteur, situation qui nous suggère que le rapport d'âge reste un élément central de la production de l'organisation sociale (Abélès et Collard 1985). Cependant, dans la hiérarchie militaire, les jeunes garçons peuvent exercer des fonctions élevées. Par exemple, le groupe rebelle Maïmaï organise la protection de ses soldats en octroyant, lors de cérémonies rituelles, des fétiches qui empêcheraient les balles d'atteindre les combattants. Lorsque les chefs considèrent que les nouvelles recrues sont prêtes à recevoir les fétiches, ils convoquent le « kadogo dawa ». Celui-ci remettra les fétiches aux concernés tout en leur enseignant les principes qui y sont associés. Le « kadogo dawa » est le plus jeune, ou l'un des plus jeunes du groupe. Il est, aux yeux des commandants, le plus fort spirituellement. Son pouvoir est saisissant : les capacités associées aux fétiches 
de l'ensemble du groupe seront détruites si la spiritualité du « kadogo dawa » est mise en péril. Par conséquent, une simple accusation de sa part auprès des commandants entraînera l'exécution des présumés coupables. Ainsi, analyser les dimensions relationnelles de la vie des ex-enfants soldats nous a permis de saisir les relations d'égalité liant adultes et enfants dans les groupes armés, l'importance du grade des militaires et la polysémie de la notion de " grand ", et enfin les bouleversements hiérarchiques de la société militaire par rapport à la hiérarchie civile».

Passons à présent aux représentations sociales des professionnels de la protection de l'enfance à propos des enfants soldats. L'anthropologie de l'enfance avait suggéré l'ambiguïté de la place des enfants soldats dans la délimitation enfant/adulte. Afin de comprendre si l'enfant soldat était un adulte ou un enfant, nous avons engagé une douzaine de personnes dans le jeu de classement exposé à la section précédente. Cet exercice, destiné à comprendre comment l'enfant soldat est défini, a in fine abouti à la conclusion selon laquelle la dualité enfance/âge adulte n'était pas ou peu classifiante. En effet, les enquêtés utilisent le critère civil/militaire pour organiser l'ensemble des portraits. À propos du groupe de militaires, il est, pour deux tiers des personnes interrogées, un bloc homogène ; et pour le troisième tiers, une catégorie sousdivisible en fonction du pouvoir ou de l'âge associé au portrait. La dualité enfant/âge adulte n'organise donc que certains classements, et de second rang. Par ailleurs, lorsque l'enfant soldat est classifié dans le groupe des militaires, les critères cités par les enquêtés regroupent alors le manque de moralité, la liberté plus grande, le sentiment de supériorité, etc. Victor, juriste kinois, argumente :

"Même cet enfant-là, on ne va pas lui demander quel âge il a, non c'est un militaire. Je le catégorise à part entière comme militaire, et on l'appelle chef quand il passe là. Même un papa va l'appeler chef ".

À défaut d'être associés aux enfants civils, les enfants soldats sont associés aux adultes soldats, principalement à travers le port d'armes et la violence commise. Nana, travailleuse sociale de rue à Kinshasa, est de cet avis. Elle argumente son opinion :

"Enfants armés, comme c'est un enfant particulier, qui a déjà une arme, ce n’est pas un enfant ordinaire. C'est un enfant avec armes. Il fera le même geste que les autres feront aussi, à se battre et tout ça. [...] Ses réactions sont autres qu'un enfant normal. Un enfant normal, à midi, il a besoin d'un bonbon, il a besoin de jouer. Celui-là a d'autres réflexes. Il sait qu'on doit être à telle position, on doit faire ça, il est soumis aux ordres auxquels il doit obéir, comme un adulte. "

Somme toute, la dualité enfant/âge adulte n'organiserait pas la perception de l'autre, et l'enfant soldat peut parfois être, pour des arguments divers, associé aux adultes militaires.

En conclusion, nos analyses d'entretiens réalisés auprès des ex-enfants soldats et les classifications opérées par les professionnels de la protection de l'enfance ont apporté divers résultats. D'une part, au sein des groupes armés, enfants et adultes entretiennent des relations de type égalitaire, le grade des militaires importe plus que leur âge, la notion de " grand » est polysémique et la hiérarchie est différente de celle d'un groupe civil. D'autre part, chez les professionnels de la protection de l'enfance, la représentation de l'autre ne se structure pas dans un premier temps sur la dualité enfance/âge adulte, et les enfants soldats sont souvent associés aux adultes militaires. Dès lors, investiguer les modalités de passation du statut de civil à celui de militaire, et inversement, nous semble plus pertinent au vu des réalités de terrain, que d'étudier les critères de démarcation de l'enfance et de l'âge adulte. Plus généralement, la notion même de passage d'un âge de la vie à un autre n'aurait pas, dans une société en conflit ou postconflit, la même pertinence que dans une société en paix. Dans ces sociétés en crise, la question de savoir si l'enfant soldat est construit socialement comme un(e) militaire ou un(e) civil(e) se substitue à la nécessité d'être instruit 
de son statut d'enfant ou d'adulte. Un proverbe en lingala, traduit ici en français, résume parfaitement nos conclusions : «La personne qui a une arme, on ne lui demande pas son âge, elle est déjà grande par son arme ».

\section{La perte du monopole de l'axe temporel}

La théorie du récit de vie met la notion de temporalité en exergue. De là, nous avons imaginé l'axe du récit, c'est-à-dire le principe organisateur, ou encore ordonnateur, des évènements " mis en intrigue " (Ricoeur 1983), comme exclusivement temporel. Or l'analyse de nos données met en évidence la présence de trois autres axes principaux dans la structuration du récit de vie : l'axe spatial, l'axe civil/ militaire et l'axe familial. Ces trois axes n'annihilent pas complètement l'axe temporel et ne s'y opposent pas simplement, mais le surpassent de loin. Nous avons analysé la présence de ces axes nouveaux en étudiant la structuration du récit, plus précisément des étapes de vie, du réseau social et des évènements divers de la vie des enquêtés.

Commençons par les étapes de vie et les types de repères que les enquêtés emploient pour les structurer. Au moyen de divers exercices " enquêteur " - " enquêtés ", d'entretiens classiques et de focus sur base de dessins, nous avons pu déceler différents types de repères biographiques. En effet, la configuration de l'exercice " enquêteur » - "enquêté » est laissée à la liberté de l'" enquêteur » ou " enquêtrice " et de l'enquêté(e) qui s'appliquent alors à utiliser les indicateurs qu'ils souhaitent. Dans ce type d'entretien, nous avons remarqué l'utilisation de deux types d'indicateurs - des indicateurs spatiaux et familiaux - qui aboutissent in fine à une orientation globale de l'entretien autour de deux axes - l'axe spatial et l'axe familial. Par exemple, certaines étapes de vie cruciales chez les ex-enfants soldats, comme l'entrée ou la sortie de la vie militaire, s'organisent, dans ce type d'entretien, autour de l'axe familial. L'extrait d'entretien suivant, qui réunit Jean et Kasama, deux ex-enfants soldats aujourd'hui démobilisés, illustre ce fait :

«Tu pensais aussi quitter l'armée ou tu souhaitais rester ? - Je souhaitais rester, mais avoir aussi cette possibilité de rendre visite à ma famille de temps à temps; mais pas de déserter. - Quand tu es parti dans l'armée, tu avais averti ta famille ou pas? - Non, je ne pouvais pas avertir, ce n'est qu'après la formation que je suis rentré pour les informer et j'ai trouvé que les parents n'étaient plus en vie. »

Par ailleurs, ces étapes paraissent également liées à la dimension spatiale. Par exemple, à la question de savoir si Papy est entré dans un groupe armé de force ou volontairement, il répondra à l'aide d'indicateurs spatiaux :

"Par rapport à la question, cette armée, nous l'avons intégrée de force. Et moi quand j'étais arrivé à Goma pour aller vers Butembo, ce n'était pas pour aller à l'armée. C'est une circonstance de la vie, qui ne m'a pas plu».

Ainsi, les étapes de vie des ex-enfants soldats se structurent majoritairement autour des axes familiaux et spatiaux. La fréquence d'indicateurs temporels est nettement moins élevée que celle d'indicateurs spatiaux ou familiaux, et souvent les questions relatives au temps sont imbriquées dans celles relatives à l'espace, comme ici : «En 2007, tu étais à Goma... ou tu étais à Kinshasa ? - Goma en 2007. En 2007 jétais ici. 2004... Non 2005 à Goma. Parce que jai quitté Gemena en 2004 et je suis allé à Kisangani " [John et Augustin]. Par ailleurs, lors des focus sur base de dessins, les enquêtés ont recours parfois à un seul, parfois à plusieurs types de repères pour structurer leurs étapes de vie. 24 personnes emploient le repère du statut de civil/militaire. Lorsqu'ils utilisent ce repère, les enquêtés organisent leurs parcours biographiques en fonction des moments où ils ont été civils, et à l'inverse, de ceux où ils ont été militaires. Ensuite, 21 personnes usent de repères spatiaux. Notons qu'il ne s'agit pas ici d'espace social 
au sens où Bourdieu l'entend dans Lillusion biographique (Bourdieu 1986:71), mais bien d'espace physique. Les espaces évoqués par les enquêtés vont du plus général - des noms de province, de ville - au plus particulier - des noms de quartier, de rue. En ce qui concerne les repères temporels, seules 11 personnes les exploitent pour marquer des étapes de leur récit de vie. Par ailleurs, si nous jetons un œil aux quelques autres repères utilisés, nous constaterons que 10 personnes se servent de repères sociaux (tel que la scolarisation, l'entrée en union, le début de la vie professionnelle, etc.), que 8 personnes s'attachent aux périodes de paix ou de guerre sévissant dans leur pays, et enfin que 6 personnes mentionnent le moment où une rupture familiale s'est produite. Au vu de ces chiffres et des indicateurs utilisés dans les exercices « enquêteurs " - « enquêtés ", nous pouvons donc faire l'hypothèse que l'axe temporel n'a pas le monopole de la structuration des étapes de vie, dont il semble pourtant jouir au niveau théorique.

Passons maintenant aux critères utilisés par l'enquêté(e) pour classer son réseau social. Nous les avons découverts grâce à 9 focus sur base de dessins. La consigne de cet exercice était la suivante : « Dessinez les personnes qui vous entourent $»$. En nous attardant sur les critères permettant aux enquêtés d'organiser en groupes les personnes qui les entourent, nous mesurons l'importance des critères notamment spatiaux et de statut civil/militaire. Le lieu de vie de l'entourage, cité par 9 enquêtés, est le critère par excellence pour organiser les personnes qui les entourent. Vient ensuite le critère de bonne/mauvaise entente qui permet à 8 personnes de classer leur entourage. Puis, 6 personnes exploitent le critère de civil/militaire et 6 autres celui de vivant/mort. Par ailleurs, le critère bon/mauvais est utilisé par 4 personnes et le type de lien avec l'enquêté(e) par 3 personnes. Le « temps » de la personne ou, en d'autres termes, la période à laquelle le réseau social se réfere, arrive en bout de course en n'étant évoqué que par une seule personne. En effet, lorsque l'enquêté(e) a à définir son entourage, il ou elle s'attache à son entourage présent, et non aux personnes qui auraient constitué son entourage dans le passé, alors que la consigne de départ n’impose pas à l'enquêté(e) de se référer à la période présente. Ainsi, d'une part, la notion de temporalité n'aurait pas d'effectivité dans la classification que les enquêtés font de leur entourage. D'autre part, les notions d'espace et de statut civil/militaire, elles, seraient des plus pertinentes.

Revenons l'espace de quelques lignes sur la manière dont ces données ont été produites. L'outil méthodologique exploité ici est le focus sur base de dessins. Pour rappel, le focus sur base de dessins a été imaginé pour déployer la narration du récit de vie sur base d'une production des enquêtés. Or, cette production, le dessin en lui-même, s'est avérée être une donnée d'enquête également, dont l'analyse aboutit aux résultats exposés ci-dessus. En devenant producteurs de données à part entière, les dessins mettent en évidence l'une des limites de l'idée de configuration narrative du récit de vie. Ainsi, si certaines cultures développent la capacité narrative des individus dès le plus jeune âge, la narration n'est pas un exercice qui va forcément de soi. Nos entretiens narratifs ont bien produit des données, mais d'autres supports non écrits se sont montrés tout aussi productifs. Le focus sur base de dessins se donne dès lors comme nouvel objectif d'être un outil de récolte de récits de vie en permettant à l'enquêteur de visualiser schématiquement de quelle manière les enquêtés répondent à une thématique.

Pour terminer, voyons comment les ex-enfants soldats font le récit d'évènements divers, présents ou passés. L'ampleur de l'influence des dimensions spatiales et familiales dans la structuration du récit se découvre ici à la fois dans l'intérêt porté au lieu de vie de l'enquêté(e) et de ses parents et dans l'évocation systématique du lieu d'un évènement quelconque. En guise d'illustration, l'extrait suivant exemplifie l'attention donnée au lieu de vie : "Pour le milieu, tu dis que tu restes à l'UPN? Oui, à l'UPN. Normalement nous ne sommes pas vraiment à l'UPN, mais plutôt sur la route qui mène vers Mont NGAFULA, arrêt LIYOLO, l'artiste qui a fait la statue de victoire » [John et Augustin]. Par ailleurs, lorsqu'ils ont à dessiner leur parcours de vie au cours des focus sur base de dessins, certains enquêtés démarrent leurs dessins par le lieu d'origine de leurs parents. C'est le cas d'Augustin, un militaire entré très jeune dans l'armée, aujourd'hui mis en disponibilité pour poursuivre ses études, et qui, en décrivant son dessin, nous dira ceci : 
«Ici, bon normalement je suis du nord Kivu. Mon père est du nord Kivu et ma mère est Mungala. Ça s'est coïncidé que mon père est venu comme administrateur du territoire en Equateur et il a rencontré ma mère. Je ne sais pas si vous me suivez ? C'est ce que j’ai montré ici. Donc c'est ce qui a fait qu'ils se rencontrent. Un moment donné après leur rencontre, ils nous ont enfantés là-bas. »

Comme nous l'avons dit, l'existence d'autres axes du récit n'annihile pas l'axe temporel. Lorsqu'elles sont mentionnées, les questions relatives au temps renvoient à la fréquence, à l'horaire, à la durée, ou encore à l'âge. En guise d'exemple, l'extrait suivant nous illustre les questions liées à l'horaire :

"Tu dis que l'ami avec qui tu vis, sort à 6 heures pour ses multiples tâches chez le colonel, et toi tu pars quand? - Moi chez le colonel, j'arrive à 8 ou 9 heures, mais s'il a vraiment besoin de moi, j'arrive très tôt vers 6 heures. - Et à l'école tu commences à quelle heure? - À 12 heures et des fois j'arrive là à 11 heures 50. - Donc tu es de l'après-midi ? - Oui. » [Papy et Justin]

\section{...qui remettent en cause les théories}

D'abord, notre recherche aboutit à la découverte, dans le parcours de vie des ex-enfants soldats, de la prépondérance de la dualité civil/militaire par rapport à la dualité enfant/adulte, et pointe ainsi du doigt le manque de pertinence de l'idée de transition de l'enfance à un âge adulte, largement véhiculée en anthropologie de l'enfance. Ensuite, les résultats de nos enquêtes ont mis en doute la pertinence de la notion de temporalité, qui semble intrinsèque à la théorie du récit de vie. Certains auteurs avaient déjà éclairé quelques lacunes de la notion en soulignant le manque de suivi chronologique du récit (Demazière 2003). Par ailleurs, l'exploitation de nos données issues des focus sur base de dessin, en remettant en cause la forme exclusivement narrative du récit de vie, renforce l'idée, déjà mentionnée par quelques auteurs (Pineau et Le Grand 1993), selon laquelle il existe d'autres moyens d'exprimer sa vie que le discours narratif.

\section{Conclusion}

Si les rapports entre théories et terrains sont controversés, ces deux sphères de la recherche ne sont pas indépendantes. L'objectif de notre article était de redorer le blason de la théorie dans l'enquête de terrain en montrant ses rôles à diverses phases de la recherche tout en explorant, au vu de nos résultats, ses limites. Nous avons ainsi d'abord exposé comment la construction de notre questionnement de recherche et par la suite, l'élaboration de notre canevas d'enquête, ont été modelées par l'anthropologie de l'enfance et la méthode du récit de vie, pour aboutir in fine à la rédaction d'une question de recherche particulière et à un échantillon et des outils de récolte de données précis. Nous avons ensuite présenté deux de nos résultats de recherche engendrés par ces mêmes questionnements et outils, pour la particularité qu'ils ont d'infirmer certains des éléments centraux de ces théories et méthodes.

D'abord, les trois notions issues de l'anthropologie de l'enfance exposées dans cet article présentent certaines limites quant à leur adéquation au domaine d'étude. Il devient bien plus important de savoir si l'enfant soldat est construit socialement comme un(e) militaire ou un(e) civil que d'être instruit de son statut d'enfant ou d'adulte. Nous souhaitons ici suggérer l'idée que la pertinence de l'idée d'une transition vers un âge adulte est fonction du type d'enfance que l'on étudie et de son contexte. Ainsi, nos résultats lèvent le voile sur certaines limites de la méthode du récit de vie. Ces limites nous invitent à proposer d'élargir la notion de récit de vie. Le récit de vie serait un ensemble complexe de données intranarratives aussi bien qu'extranarratives organisées autour d'axes spatiaux, familiaux, de statuts civil/ militaire, ou temporels selon le contexte de recherche et la population visée par l'étude. 
En conclusion, d'après les apports respectifs de l'anthropologie de l'enfance et du récit de vie, nous pouvons conclure que la théorie façonne le terrain en modelant la construction du questionnement de recherche et l'élaboration du canevas d'enquête. Ensuite de quoi les limites mises à jour par certaines analyses de données encouragent le chercheur à proposer des aménagements, ou des approfondissements des concepts. De sorte que nous sommes amenée à conclure que, réciproquement, le terrain lui aussi façonne la théorie. Ainsi, les rapports entre théorie et terrain ne sont pas univoques, unilinéaires et irréversibles. Le va-et-vient entre ces deux entités présentes dans toute recherche socio-anthropologique est vital. Et si notre article met en évidence ce double façonnement, ce n'est pas dans le but de juger de la pertinence de notions théoriques, mais bien dans le souci de dégager la nécessaire itération abstraite (Olivier de Sardan 2008:82) entre théories et terrains.

\section{Note}

1. Les images utilisées ont été téléchargées à partir de différentes banques d'images publiques disponibles sur Internet.

\section{Références}

Abélès, M. et Collard, C., 1985, Âge, pouvoir et société en Afrique noire, Paris, Karthala.

Antoine, P., Razafindrakoto, M. et Roubaud, F., 2001, 'Contraints de rester jeunes ? Évolution de l'insertion dans trois capitales africaines : Dakar, Yaoundé, Antananarivo', in Diouf, M. et Collignon, R., ed.., Les jeunes, hantise de l'espace public dans les sociétés du Sud ?, Editions de l'Aube, IRD, Autrepart, No 18, p. 17-36.

Badie, B., 2006, L'État importé, l'Occidentalisation de l'ordre politique, Paris, Fayard.

Balandier, G., 2001, Le grand système, Paris, Fayard.

Bayart, J.-F., 1990, La problématique de la démocratie en Afrique noire, La Baule, et puis après ?, document de travail rédigé en juin 1990, à l'approche de la conférence de La Baule.

Bertaux, D., 1997, Les récits de vie, Paris, Nathan.

Biaya, T. K., 2001, 'Les plaisirs de la ville : Masculinité, sexualité et féminité à Dakar (19972000)', African studies review, Vol. 44, N², p. 71-85.

Bourdieu, P., 1986, 'Lillusion biographique', Actes de la recherche en sciences sociales, Vol. 62-63, p. 6972.

Boyden, J., 1997, 'Childhood and the Policy Makers : a Perspective on the Globalization of Childhood', in James, A. et Prout, A., ed., Constructing and Reconstructing Childhood : contemporary issues in the sociological study of childhood, Cambridge, Falmer Press, p. 190-229.

Burrick, D., 2010, 'Une épistémologie du récit de vie', Recherches qualitatives, № 8, p. 7-36.

D’Almeida-Topor, H., Coquery-Vidrovitch, C. et Goerg O., 1992, Les jeunes en Afrique. La politique et la ville, Vol. 2, Paris, L'Harmattan.

Danic, I., Delalande, J. et Rayou, P., 2006, Enquêter auprès d'enfants et de jeunes. Objets, méthodes et terrains de recherche en sciences sociales, Presses Universitaires de Rennes.

Darbon D., du Bois de Gauduson, J., ed., 1997, La création du droit en Afrique, Paris, Karthala.

De Boeck, F., 2005, Kinshasa, Récits de la ville invisible, Paris, La Renaissance du Livre.

De Boeck, F. et Honwana, A., 2005, Makers and Breakers. Children and Youth in Post-Colonial Africa, Oxford, James Currey.

De Gaujelac, V., 1987, La névrose de classe : trajectoire sociale et conflits d'identité, Paris, Hommes et Groupes.

Delory-Momberger, C., 2005, Histoire de vie et recherche biographique en éducation, Paris, Economica. 
Demazière, D., 2003, 'Matériaux qualitatifs et perspectives longitudinales. La temporalité des parcours professionnels saisie par les entretiens biographiques', 10e journée sur les données longitudinales dans l'analyse du marché du travail, Caen, LASMAS-CEREQ.

Demazière, D. et Dubar, C., 2009, Analyser les entretiens biographiques. L'exemple de récits de réinsertion, Presses de l'Université de Laval.

Denov, M., 2010, Child soldiers, Sierra Leone's Revolutionary United Front, Cambridge University Press.

De Suremain, C.-E., 2006, 'Affinités horizontales et stratégies de survie parmi les enfants de la rue. La bande Solitarios à La Paz (Bolivie)', Tiers monde, $N^{\circ} 185$, p. 113-133.

Fall, A.-S. et Massart, G., 2007, 'Sur les traces des enfants et des jeunes ouest-africains en faveur d'une intervention d'écoute active', Plan international.

Garnier, P., 1995, Ce dont les enfants sont capables. Marcher, travailler, nager. XVIIe XIXe XXe siècles, Paris, Ed. Métaillé.

Goodwin-Gill, G. et Cohn, I., 1994, Child soldiers, the role of children in armed conflicts, Oxford, Clarendon Press.

Gretry, L., 2011, 'Child soldiers : our representation challenged by their reality', International journal of sociology and social policy, Vol. 31, Nos. 9\&10, p. 583-593.

Guillaume, J.-F., Lalive d'Epinay C. et Thomsin, L., 2005, Parcours de vie. Regards croisés sur la construction des biographies contemporaines, Les éditions de l'université de Liège.

Hart, J., 2006, 'The Politics of Child Soldiers', Brown Journal of World Affairs, Vol. 13, N 1, p. 217226.

Honwana, A., 2000, 'Innocents et coupables à la fois : les enfants soldats comme acteurs tactiques', in De Boeck, F. et Honwana, A., ed.., Enfants, jeunes et politiques, Paris, Politique africaine, $\mathrm{N}^{\circ} 80$, p. $58-78$.

Hoyles, M., 1979, Changing Childhood, London, Writers and Readers Pub., Coop.

Jenks, C., 1996, Childhood, Key Ideas Series, London and New York, Routledge.

Jézéquel, J.-H., 2006, 'Les enfants soldats d'Afrique, un phénomène singulier' Vingtième siècle, № 89, p. $99-108$.

Kaufmann, J.-C., 2004, L'invention de soi : une théorie de l'identité, Paris, Nathan Université.

Krueger, R.-A., 1994, Focus Group. A practical guide for applied research, London, Sage.

Kuyu, C., 2008, Ecrits d'anthropologie juridique et politique, Louvain-la-neuve, Bruylant-Academia s.a.

Lainé, A., 2008, 'Lapproche « roman familial et trajectoire sociale », in De Gaujelac, V. et Legrand, M., ed.., Intervenir par le récit de vie : entre histoire collective et histoire individuelle, Ramonville SainteAgne, Erès, p. 149-168.

Legrand, M., 1993, L’approche biographique. Théorie, clinique, Paris, éditions Presses Internationales.

Le Grand, J.-L., 2000, 'Définir les histoires de vie', Revue internationale de psychologie, Vol. 14, p. 29-46.

Lévesque, J.-L., 2003, 'Il était une fois... le récit de vie', in Leahey, J. et Yelle, C., ed.., Histoires de liens, histoires de vie. Lier, délier, relier, Paris, L'Harmattan, p. 71-86.

Lucchini, R., 1996, Sociologie de la survie : l'enfant dans la rue, Paris, PUF.

Machel, G., 1996, «Impact of Armed Conflict on Children » (UN Doc. A/51/306). Available online at : http://www.unicef.org/graca/a51-306_en.pdf (accessed 20 October 2011).

Marie, A., 1997, L’Afrique des individus, Paris, Karthala.

Mbembe, J.-A., 2000, De la postcolonie : Essai sur l'imagination politique dans l'Afrique contemporaine, Paris, Karthala.

Mouzayan, O.-H., 2003, L'enfant-soldat, Paris, Odile Jacob. 
Muxel, A. et Cacouault, M., 2001, Les jeunes d'Europe du Sud et la politique. Une enquête comparative France, Italie, Espagne, Paris, L'Harmattan.

Neyzi, L., 2001, 'Object or Subject ? The paradox of « Youth » in Turkey', in Diouf, M. et Collignon, R., ed.., Les jeunes, hantise de l'espace public dans les sociétés du Sud ?, Autrepart, N ${ }^{\circ} 18$.

Olivier de Sardan, J.-P., 2008, La rigueur du qualitatif, les contraintes empiriques de l'interprétation socio-anthropologique, Louvain-la-Neuve, Academia Bruylant.

Orofiamma, R., 2002, 'Le travail de la narration dans le récit de vie', in Niewiadomski, C. et de Villers, G., Souci et soin de soi, Liens et frontières entre histoire de vie, psychothérapie et psychanalyse, Paris, L'Harmattan.

Orofiamma, R., 2008, 'Les figures du sujet dans le récit de vie, En sociologie et en formation', CNAF Informations sociales, Vol. 1, No 145, p. 68-81.

Pineau, G. et Le Grand J.-L., 1993, Les histoires de vie, PUF.

Poirier, J., Clapier-Valladon, S. et Raybaut, P., 1989, Les récits de vie. Théories et pratiques, PUF.

Ricoeur, P., 1983, Temps et récit. Tome I, L'intrigue et le temps historique, Paris, éditions du Seuil.

Rosen, M., 2005, Armies of the Young : Child Soldiers in War and Terrorism, Rutgers University Press.

Singer, P., 2005, Children at War, New York, Pantheon Books.

Sirota, R., 2006, Éléments pour une sociologie de l'enfance, Presses Universitaires de Rennes.

Tatek, A., 2008, Ethiopian Childhoods, A Case Study of the Lives of Orphans and Working Children, Doctoral thesis at NTNU.

Tessier, S., 1995, Langages et cultures des enfants de la rue, Paris, Karthala.

Trefon, T., 2004, Ordre et désordre à Kinshasa, réponses populaires à la faillite de l'Etat, Cahiers Africains. 